\title{
An unusual malposition of central venous catheter in critically ill patient. Could it be identified during catheterization? The potential role of point-of-care ultrasonography.
}

\author{
Gentle Sunder Shrestha ${ }^{1}$, Bishesh Sharma Poudyal ${ }^{2}$
}

\begin{abstract}
Malpositioning of central venous catheter is not uncommon and can be associated with complications. We report a case where central venous catheter inserted through right internal jugular approach under real time ultrasonographic guidance was malpositioned with its tip in left brachio-cephalic vein. Chest radiograph for detection of catheter malpositioning is associated with time delay and radiation exposure. Point-of-care ultrasonography can be a valuable bedside tool to identify catheter malpositioning during catheter insertion, thus facilitating immediate catheter repositioning and early use of central venous catheter.
\end{abstract}

Key Words: central venous catheter, malposition, point-of-care ultrasonography

\section{Introduction :}

Central venous catheters (CVCs) are inserted in ICU for a variety of indications like administering drugs (vasopressors/inotropes, antibiotics, chemotherapy), monitoring central venous pressure (CVP), measurement of central venous oxygen saturation, renal replacement therapy, total parenteral nutrition, poor peripheral venous access, cardiac catheterization and transvenous cardiac pacing. ${ }^{1}$ Successful CVC placement includes positioning of the catheter tip near the right atrium. The optimal CVC tip placement using the subclavian and internal jugular venous approaches is in the distal superior venacava above the preicardial reflection. ${ }^{2}$ Incidence of CVC malposition ranges from 3.6 to $14 \%$. Cannulation by the right subclavian vein is associated with the highest risk of malposition. ${ }^{3}$

Here we report a rare CVC malposition in a critically ill patient with septic shock. The tip of catheter inserted through right internal jugular approach was in left brachiocephalic vein.

American College of Radiology recommends chest radiograph after insertion of $\mathrm{CVC}$ to demonstrate proper placement and detect any complications. ${ }^{4}$ However, performance and interpretation of the post-CVC chest

1. Dr. Gentle Sunder Shrestha ${ }^{1}$, Neuro-Intensivist and Anaesthesiologist Institute of Medicine, Tribhuvan University Teaching Hospital

2. Dr. Bishesh Sharma Poudyal, Clinical Haematologist and Bone marrow Transplant Physician. Department of Internal Medicine, Civil Service Hospital, Kathmandu, Nepal.

Corresponding Author:

Dr. Gentle Sunder Shrestha

Neuro-Intensivist and Anaesthesiologist

MD, FACC, EDIC, FCCP

Lecturer, Department of Anaesthesiology,

Institute of Medicine

Tribhuvan University Teaching Hospital

Maharajgunj, Kathmandu, Nepal

Email: gentlesunder@hotmail.com radiography is associated with time delay, additional radiationexposure and additional cost. ${ }^{2}$ Early goal-directed therapy provides significant benefit with respect to outcome in patients with severe sepsis and septic shock. ${ }^{5}$ Early confirmation of proper CVC position and use of CVC can facilitate institution of early goal-directed therapy.

Various bed side modalities like point-of-care ultrasonography, ${ }^{2,6}$ real-time X-ray imaging, ${ }^{7}$ pressure waveform analysis ${ }^{1}$ and ECG guidance ${ }^{8}$ can be helpful to detect malposition at the time of catheterization.

\section{Case Description :}

A 23 years old lady was admitted to ICU with the diagnosis of septic shock due to severe community acquired pneumonia. She was a known case of Acute Myeloid Leukemia. Central venous access was planned for administration of vasopressors and for monitoring of central venous pressure. She was thrombocytopenic with platelet count of $45,000 / \mathrm{cu} \mathrm{mm}$.

Central venous catheter was inserted through right internal jugular approach under real time ultrasonographic guidance. Cannulation was done after confirmation of guidewire in the vein by ultrasound. Catheter was secured at $13 \mathrm{~cm}$ after aspiration of blood from all the lumens. Procedure was uneventful and was performed in a single attempt. Central venous pressure, measured manually was $21 \mathrm{~cm}$ of $\mathrm{H}_{2} \mathrm{O}$. Portable chest X-ray revealed the catheter tip malpositioned in left brachio-cephalic vein (Fig. 1.). The catheter was withdrawn upto $8 \mathrm{~cm}$ and was resinserted over the guidewire. Repeated chest X-ray revealed proper position of catheter in lower superior venacava. 


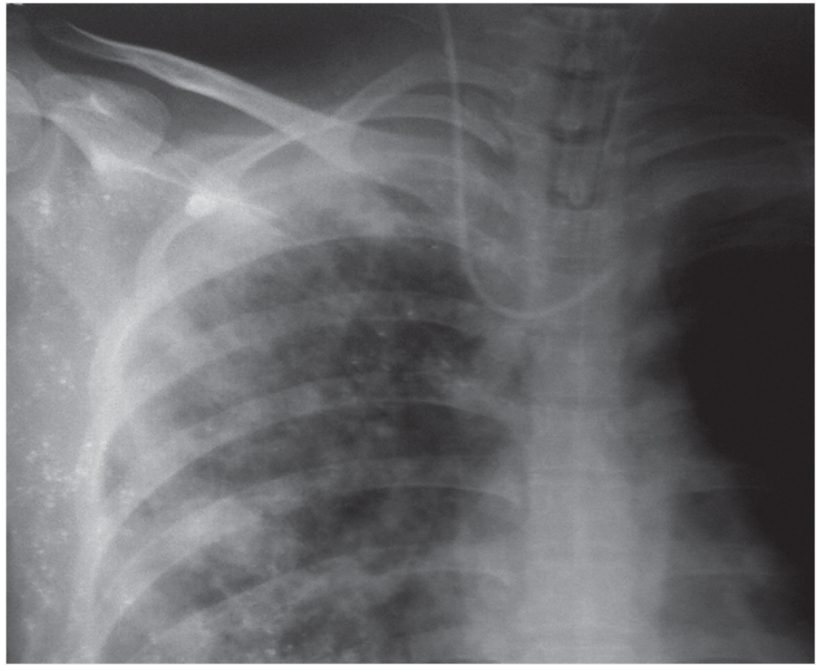

Fig. 1: Central venous catheter inserted through right internal jugual approach. Catheter tip malpositioned in left brachio-cephalic vein (indicated by arrow).

\section{Discussion :}

CVC malposition can be associated with the complications like inaccurate CVP monitoring, potential irritation of the valves by catheter or infused fluids, vessel thrombosis and cardiac tamponade. ${ }^{1}$ Though recommended by guidelines, ${ }^{4}$ post CVC chest radiography has limitations, due to their 2D projection. Close anatomical proximity with major arteries, veins and pleura in the neck and chest makes it not possible to reliably state whether the distal section of the catheter is in an artery, vein, pleura, or mediastinum from a plain chest X-ray. ${ }^{1}$ Subsequent repositioning of catheter is not free of risk. The guidewire repositioning of the catheter may be associated with a greater risk of catheter-related infection. ${ }^{9}$ In our case, guidewire repositioning of the malpositioned catheter was performed, as the patient was at risk of mechanical complications if inserted at new site, due to existing thrombocytopenia.

Real-time X-ray imaging using an image intensifier during CVC placement has similar limitations to a plain chest X-ray. ${ }^{7}$ Intravascular ECG monitoring is helpful to verify central placement of catheter within the chest at the caval atrial junction, ${ }^{8}$ but it is not useful for differentiating between venous and arterial placement of CVC. ${ }^{10}$

Bedside point-of-care ultrasonography using saline flush ${ }^{2}$ or contrast enhanced ultrasonography ${ }^{6}$ was shown to be the reilable screening test for optimal CVC tip position. Rapid appearance of prominent turbulence in the right atrium on echocardiography after CVC saline flush reliably identified proper CVC tip position. ${ }^{2}$ Combination of B-mode ultrasonography and contrast enhanced ultrasonography reliably detected catheter misplacement. Bedside ultrasonography was also valuable in detecting pneumothorax. Time required for sonography was significantly shorter than for chest radiograph. ${ }^{6}$
To conclude, like in our patient, use of point-of-care ultrasonography potentially could have identified catheter malposition during catheterization, thus expediting the catheter repositioning and prompt institution of early goal direct therapy.

\section{References :}

1. Gibson F, Bodenham A. Misplaced central venous catheters: applied anatomy and practical management. $\mathrm{Br} J$ Anaesth 2013;110:333-46.

2. Weekes AJ, Johnson DA, Keller SM, Efune B, Carey C, Rozario NL, et al. Central vascular catheter placement evaluation using saline flush and bedside echocardiography. Acad Emerg Med 2014;21:65-72.

3. Pikwer A, Baath L, Davidson B, Perstoft I, Akeson J. The incidence and risk of central venous catheter malpositioning: a prospective cohort study in 1619 patients. Anaesth Intensive Care 2008;36:30-7.

4. Amorosa JK, Bramwit MP, Mohammed TL, Reddy GP, Brown K, Dyer DS, et al. ACR appropriateness criteria routine chest radiographs in intensive care unit patients. $\mathrm{J} \mathrm{Am}$ Coll Radiol 2013;10:170-4.

5. Rivers E, Nguyen B, Havstad S, Ressler J, Muzzin A, Knoblich B, et al. Early goal-directed therapy in the treatment of severe sepsis and septic shock. N Engl J Med 2001;345:1368-77.

6. Vezzani A, Brusasco C, Palermo S, Launo C, Mergoni M, Corradi F. Ultrasound localization of central vein catheter and detection of postprocedural pneumothorax: an alternative to chest radiography. Crit Care Med 2010;38:533-8.

7. Tan PL, Gibson M. Central venous catheters: the role of radiology. Clin Radiol 2006;61:13-22.

8. McGee WT, Ackerman BL, Rouben LR, Prasad VM, Bandi V, Mallory DL. Accurate placement of central venous catheters: a prospective, randomized, multicenter trial. Crit Care Med 1993;21:1118-23.

9. Cook D, Randolph A, Kernerman P, Cupido C, King D, Soukup C, et al. Central venous catheter replacement strategies: a systemic review of the literatures. Crit Care Med 1997;25:1417-24.

10. Schummer W, Schummer C, Paxian M, Frober R, Settmacher U. ECG recording of central venous catheter misplaced in inferior thyroid artery. Br J Anaesth 2005;94:296-9. 